\title{
NEW DATABASE PROVIDES INSIGHT INTO EUROPEAN COUNTRIES' SUBSPECIALTY TRAINING IN NEONATOLOGY
}

\author{
M. Breindahl ${ }^{1}$, M. Blennow ${ }^{2}$, M.T. Lluch ${ }^{3}$, J.-C. Fauchere ${ }^{4}$, J.-C. Picaud ${ }^{5}$, G. Greisen ${ }^{1}$ \\ ${ }^{1}$ Department of Neonatology, Rigshospitalet, Copenhagen University Hospital, Copenhagen, Denmark, \\ ${ }^{2}$ Department of Neonatology, Karolinska Institutet and University Hospital, Stockholm, Sweden, \\ ${ }^{3}$ Department of Neonatology, Hospital Sant Joan de Deu, Universitat de Barcelona, Barcelona, Spain, \\ ${ }^{4}$ Perinatal Center, Division of Neonatology, University Hospital, Zurich, Switzerland, ${ }^{5}$ Service de \\ Néonatologie et Réanimation Néonatale, Hopital de La Croix Rousse, Lyon, France
}

Background and aims: The ESN decided to establish a database of the European countries' national programs on subspecialty training in Neonatology. The aim was to improve transparency and facilitate harmonisation of standards of knowledge and skills required to practice Neonatology at a tertiary care level. The process was undertaken January, 2010 - March, 2011.

Methods: Based on the ESN Curriculum for Training in Neonatology (Syllabus) we constructed a database containing 6 categories according to the Syllabus: Training-knowledge, basic characteristics, training-skills, key competencies, personal development, and recording of progress. UEMS (Union of European Medical Societies) member countries were contacted by e-mail, and relevant contact persons were identified. The majority of data was categorical, i.e. "yes", "no" or "not applicable". Basic characteristics concerning e.g. length of training in Neonatology were quantified.

Results: A total of 28/30 UEMS member countries (93\%) completed the database which was published on the ESN website. We found high uniformity across countries concerning the knowledge, skills and competencies required of a trainee. However, quality control measures mentioned under basic characteristics such as examination, confrontations and allocation of a mentor were extremely different between responders. The same was true for the responders' expectations to the trainee's personal development as counsellor, manager, leader and teacher, as well as recording of progress.

Conclusions: This database provides the first overview of the European countries' national training programs in Neonatology. We hope that the transparency generated can serve to identify differences that are founded in local conditions and those that can be harmonised. 